\title{
Impact of Water Coadsorption on the Electrode Potential of H-Pt(1 1 1)-Liquid Water Interfaces
}

\author{
Sudarsan Surendralal $\odot,{ }^{*}$ Mira Todorova $\odot,{ }^{\dagger}$ and Jörg Neugebauer $\odot$ \\ Department of Computational Materials Design, Max-Planck-Institut für Eisenforschung GmbH, \\ Max-Planck-Straße 1, D-40237 Düsseldorf, Germany
}

(Received 22 October 2020; revised 10 February 2021; accepted 30 March 2021; published 21 April 2021)

\begin{abstract}
Density functional theory molecular dynamics simulations of $\mathrm{H}$-covered $\mathrm{Pt}(111)-\mathrm{H}_{2} \mathrm{O}$ interfaces reveal that, in contrast to common understanding, $\mathrm{H}_{2} \mathrm{O}$ coadsorption has a significant impact on the electrode potential of and plays a major role in determining the stability of $\mathrm{H}$ adsorbates under electrochemical conditions. Based on these insights, we explain the origin behind the experimentally observed upper limit of $\mathrm{H}$ coverage well below one monolayer and derive a chemically intuitive model for metal-water bonding that explains an unexpectedly large interaction between coadsorbed water and adsorbates.
\end{abstract}

DOI: 10.1103/PhysRevLett.126.166802

Adsorption of ions at electrode-electrolyte interfaces controls the electrode potential as well as the rate of electrochemical reactions relevant to electrochemical energy storage, electrocatalysis, and aqueous corrosion. While the relation between the electrode potential and adsorbate coverage is accessible by experimental techniques such as cyclic voltammetry [1-5], understanding how exactly adsorbate interfacial structures influence the measured electrode potential evades direct experimental access. In contrast, for surfaces in vacuum, determining the adsorbate structure is possible via modern surface science techniques [6-10]. Also, at surface science conditions the work function, which is formally equivalent to the electrode potential $[11,12]$, is experimentally straightforward to measure. A comparison of key experimental data such as the voltage-coverage dependence or adsorption energies obtained from surface science techniques and in an electrochemical setup often show very similar behavior. Based on these empirical observations, a common assumption is that adsorbate binding, as well as adsorbate-adsorbate structure and interactions, can be accurately approximated from surface science experiments or surface science modeling [6,13-21].

Surface science models, however, have been unable to explain some important properties of metal-water interfaces. Consider the $\mathrm{H}$-covered $\mathrm{Pt}(111) / \mathrm{H}_{2} \mathrm{O}$ interface, which is also one of the best investigated "model" electrochemical systems $[1,3,4,22-36]$ due to its excellent

Published by the American Physical Society under the terms of the Creative Commons Attribution 4.0 International license. Further distribution of this work must maintain attribution to the author(s) and the published article's title, journal citation, and DOI. Open access publication funded by the Max Planck Society. catalytic performance for the hydrogen evolution reaction (HER). While surface science principles have been used to successfully reproduce voltammograms and the voltagecoverage dependence for this system [4,5], they fail to explain a key experimental observation: The presence of an upper limit to the $\mathrm{H}$ adsorption at $\approx 0.66 \mathrm{ML}$ (monolayer). This upper limit also coincides with the onset of the HER $[2,3]$. A possible explanation is that water is not just a spectator but actively controls adsorption and chemical reactions at the metal-water interface.

To understand the impact of water on surface adsorption and the electrode potential, we performed a systematic comparison of the work function (electrode potential) of $\mathrm{H}$-covered $\mathrm{Pt}\left(\begin{array}{lll}1 & 1 & 1\end{array}\right)$ with $\mathrm{H}$ coverages $\left(\Theta_{\mathrm{H}}\right)$ of up to a single monolayer in vacuum and an aqueous environment employing density functional theory (DFT) calculations using the Vienna ab initio simulation package [37-39]. The equilibrium adsorbate structures and the work function values for the surfaces in vacuum are obtained after structural optimization, while DFT molecular dynamics (DFT-MD) simulations with explicit water molecules confined between the $\mathrm{Pt}$ surface and a computational $\mathrm{Ne}$ counter electrode based on our recently developed computational approach [40] are used to sample structures and their corresponding electrode potential values in the presence of water [41].

The calculated work function and electrode potential values are summarized in Fig. 1. A comparison with the experimental data for the surface in vacuum [18] (solid black line) shows an excellent agreement with our DFT computed results (blue line). For the system in contact with water, there is a similar agreement with the experimental data (black crosses) obtained from cyclic voltammetry $[2,56]$ for $\Theta_{\mathrm{H}}<0.66 \mathrm{ML}$. At these coverages, the work function and electrode potential values show the same trend in experiment and in our computational work: 


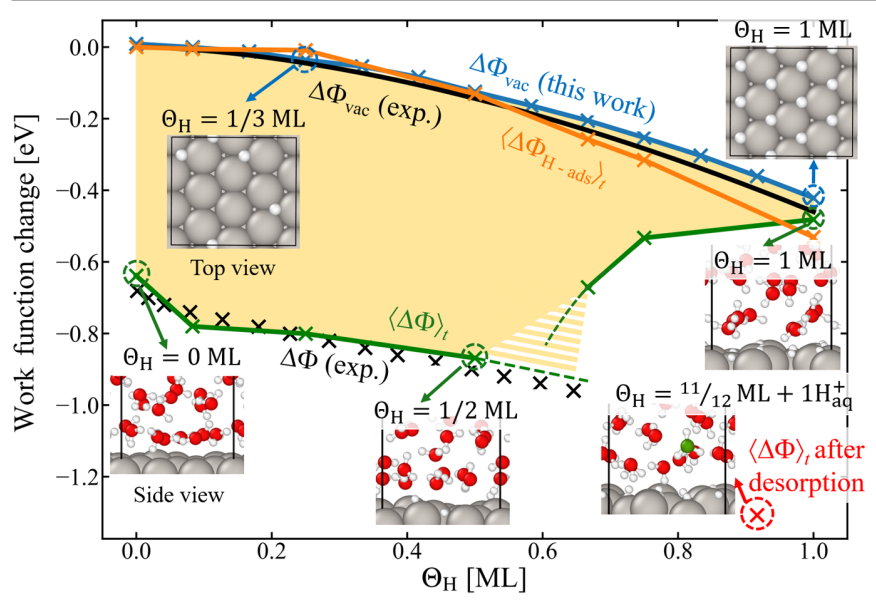

FIG. 1. Work function and time-averaged electrode potential as function of $\mathrm{H}$ coverage $\left(\Theta_{\mathrm{H}}\right)$ for the $\operatorname{Pt}\left(\begin{array}{lll}1 & 1 & 1\end{array}\right)$ surface in vacuum $\left(\Delta \Phi_{\mathrm{vac}}\right)$ and in contact with water $\left(\langle\Delta \Phi\rangle_{t}\right)$. For comparison, the experimentally measured work function [18] and electrode potential [2] are included. All potentials are referenced to the work function of the clean (adsorbate free) $\operatorname{Pt}\left(\begin{array}{lll}1 & 1 & 1\end{array}\right)$ surface. Additionally, the $\mathrm{H}$ adsorbate induced change in the work function $\left\langle\Delta \Phi_{\mathrm{H} \text {-ads }}\right\rangle_{t}$ for the system in the aqueous phase (see text) is also plotted here. The yellow hashed area lies between two DFT computed concentrations and marks the region where the potential minimum (i.e., the critical $\mathrm{H}$ concentration) is predicted. The dashed green lines extrapolate the low and high H-coverage case in this region. Representative surface and interface structures at select coverages are shown as insets. Gray, white, red, and green spheres represent $\mathrm{Pt}, \mathrm{H}, \mathrm{O}$ of $\mathrm{H}_{2} \mathrm{O}$ molecules and $\mathrm{O}$ of the solvated proton, respectively. The black line shows the simulation cell boundary.

they decrease with increasing $\mathrm{H}$ coverage. While for surface science conditions this trend monotonously extends to a full hydrogen ML, under aqueous conditions the adsorbate induced monotonous lowering in the potential stops abruptly before a full ML coverage is reached. The corresponding critical coverage in experiment is $\Theta_{\mathrm{H}} \approx 0.66 \mathrm{ML}$. Since the DFT computations can be only performed for a rather small set of $\mathrm{H}$ concentrations, the critical coverage cannot be exactly determined but is given by the interval between two data points. As shown by the yellow-shaded area in Fig. 1, this interval is $0.5 \mathrm{ML}<\Theta_{\mathrm{H}}<0.66 \mathrm{ML}$, i.e., the upper bound coincides with the experimental value.

These results can be interpreted as follows: in the lowcoverage region, the system can respond to lowering of the electrode potential by increasing the $\mathrm{H}$ coverage. This direct connection between $\mathrm{H}$ coverage and electrode potential breaks down at $\Theta_{\mathrm{H}}<0.66 \mathrm{ML}$ (in the calculations) and at $\Theta_{\mathrm{H}} \approx 0.66 \mathrm{ML}$ (in experiment). Above this point, a further increase in $\mathrm{H}$ adsorption will no longer result in a decrease in the electrode potential. A way for the system to respond to the application of these large negative potentials is by creating a large interfacial dipole through an electrochemical desorption reaction. Indeed, as shown in
Fig. 1, the full ML adsorbate structure is only metastable, and after $\approx 25 \mathrm{ps}$, one of the $\mathrm{H}$ adatoms $\left(\mathrm{H}_{\mathrm{ad}}\right)$ desorbs, resulting in a 11/12 ML $\mathrm{H}$ surface coverage and a single proton $\mathrm{H}_{\mathrm{aq}}^{+}$in solution $\left(\mathrm{H}_{\mathrm{ad}} \rightarrow \mathrm{H}_{\mathrm{aq}}^{+}+\mathrm{e}^{-}\right)$. This step provides the necessary conditions for the HER. The electrode potential for the desorbed state, as expected, is significantly lower than any potential that can be achieved by $\mathrm{H}$ adsorption alone. The above discussion directly links the existence of the breakdown in the electrode potential and the experimentally observed critical $\mathrm{H}$ adsorbate concentration at which hydrogen evolution starts. Based on this insight, two questions arise: first, what is the origin of the breakdown, and second, why is it absent in the waterfree case?

To address these questions, we decompose the change in the electrode potential into three contributions:

$$
\Delta \Phi=\Delta \Phi_{\mathrm{H} \text {-ads }}+\Delta \Phi_{\text {elec }}+\Delta \Phi_{\text {orient }} .
$$

The first contribution, $\Delta \Phi_{\mathrm{H}-\mathrm{ads}}$, describes the contributions related to $\mathrm{H}$ adsorption and is conceptually equivalent to the mechanism present in vacuum. It is computed by averaging over a set of uncorrelated snapshots from our molecular dynamics (MD) runs sampled at least $0.5 \mathrm{ps}$ apart,

$$
\left\langle\Delta \Phi_{\mathrm{H}-\mathrm{ads}}\right\rangle_{t}=\frac{\sum_{i=1}^{N} \Phi_{\mathrm{Pt} \mid \mathrm{H}}^{i}-\Phi_{\mathrm{Pt}}^{i}}{N}
$$

Here, $i$ is the index running over the $N$ uncorrelated snapshots. $\Phi_{\mathrm{Pt} \mid \mathrm{H}}$ and $\Phi_{\mathrm{Pt}}$ are the respective work functions of the system $\mathrm{Pt}$ with $\mathrm{H}$ adsorbates and the system with $\mathrm{Pt}$ atoms only. For both cases, the water molecules and the counter electrode have been removed from each snapshot. This approach is identical to the one we use to determine the work function for the vacuum surfaces except that we do not perform structural energy minimization but use the frozen in structures from the MD simulations.

The second and third contributions occur only in an aqueous environment. The second contribution, $\Delta \Phi_{\text {elec }}$, stems from electronic charge transfer between metal and water. It is again a time-averaged quantity calculated using the DFT-MD snapshots. It is obtained from the charge density of the snapshot with only $\mathrm{Pt}$ and $\mathrm{H}$ adsorbates $n_{\mathrm{Pt} \mid \mathrm{H}}(\mathbf{r})$, and with only water and the Ne counter electrode $n_{\mathrm{H}_{2} \mathrm{O} \mid \mathrm{Ne}}(\mathbf{r})$. These are subtracted from the charge density of the snapshot of the entire interface $n_{\mathrm{Pt}|\mathrm{H}| \mathrm{H}_{2} \mathrm{O} \mid \mathrm{Ne}}(\mathbf{r})$ to get the difference charge density

$$
\Delta n(\mathbf{r})=n_{\mathrm{Pt}|\mathrm{H}| \mathrm{H}_{2} \mathrm{O} \mid \mathrm{Ne}}(\mathbf{r})-n_{\mathrm{Pt} \mid \mathrm{H}}(\mathbf{r})-n_{\mathrm{H}_{2} \mathrm{O} \mid \mathrm{Ne}}(\mathbf{r}) .
$$

This difference includes any charge transfer between water and the slab, as well as electronic polarization induced by the slab into water and vice versa. The potential obtained by solving the Poisson equation for this difference 

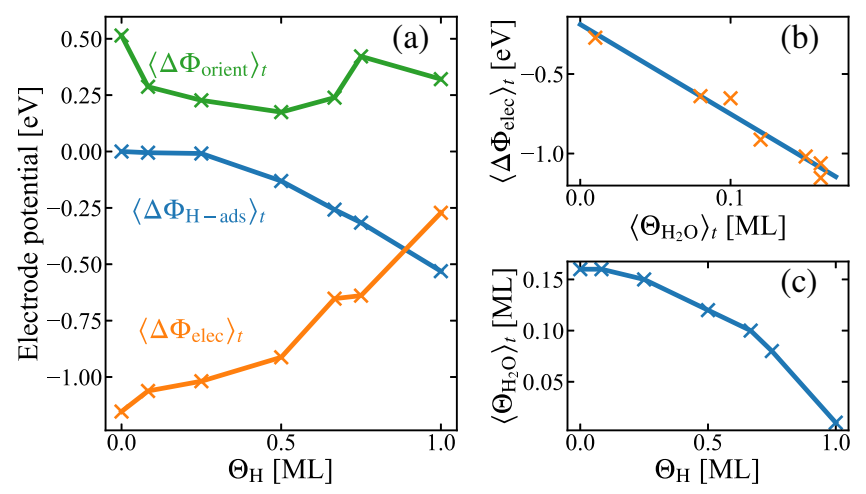

$\left\langle\Theta_{\mathrm{H}_{2} \mathrm{O}}\right\rangle_{t}[\mathrm{ML}]$

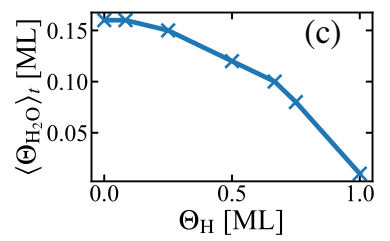

FIG. 2. (a) Decomposition of the electrode potential vs $\mathrm{H}$ coverage according to Eq. (1). (b) Correlation between the electronic contribution of the potential $\Delta \Phi_{\text {elec }}$ and chemisorbed water coverage $\Theta_{\mathrm{H}_{2} \mathrm{O}}$ where the blue line shows the linear regression. (c) Chemisorbed water coverage (see text) $\Theta_{\mathrm{H}_{2} \mathrm{O}}$ vs $\Theta_{\mathrm{H}}$

charge density provides direct access to $\Delta \Phi_{\text {elec }}$ (for more details, see the Supplemental Material [41]).

The third contribution, $\Delta \Phi_{\text {orient }}$, is due to the reorientation polarization of water molecules in the vicinity of the interface. It is obtained from Eq. (1) as the difference of the total potential drop minus the first two contributions. It describes the reorientation of the water molecules to screen the electric field [57].

These contributions are plotted in Fig. 2(a), and their values are listed in Table I of the Supplemental Material [41].

Considering only the contribution related to H-adsorption $\Delta \Phi_{\mathrm{H}-\text { ads }}$ [plotted in Figs. 1 and 2(a)], a qualitatively similar dependence on $\mathrm{H}$-coverage $\Theta_{\mathrm{H}}$ is observed as in the vacuum case: the potential monotonously decreases with increasing $\mathrm{H}$ coverage. This result may appear to support the surface science picture that the impact of water is small. However, consider the two water-related contributions, $\Delta \Phi_{\text {elec }}$ and $\Delta \Phi_{\text {orient }}$, in Fig. 2(a): their magnitude is similar or even larger than $\Delta \Phi_{\mathrm{H}-\mathrm{ads}}$. A closer inspection shows that, for coverages up to about half a monolayer, the two waterrelated contributions have an approximately opposite dependence on $\mathrm{H}$ coverage. Consequently, for lower $\mathrm{H}$ coverages, work function and electrode potential values are shifted but show a rather similar coverage dependence (Fig. 1). For $\mathrm{H}$ concentrations above $0.5 \mathrm{ML}$, the ability to further lower the potential by water reorientation is exhausted. Thus, this mechanism is no longer able to further compensate for the steadily increasing $\Delta \Phi_{\text {elec }}$. This is the source of the voltage breakdown discussed above and the origin of an upper limit for $\mathrm{H}$ adsorption well below 1 ML.

To understand why $\Delta \Phi_{\text {elec }}$ steadily increases with increasing $\mathrm{H}$ coverage, we have analyzed in detail the DFT-MD trajectories. We find that water molecules interact with the $\operatorname{Pt}\left(\begin{array}{lll}1 & 1 & 1\end{array}\right)$ surface as chemisorbed and physisorbed adsorbates with Pt-O bond lengths of 2.3-2.4 $\AA$ and 3.3-3.6 A, respectively. A further analysis shown in Fig. 2(b) reveals that the electronic component $\Delta \Phi_{\text {elec }}$ correlates almost linearly with the coverage $\left(\Theta_{\mathrm{H}_{2} \mathrm{O}}\right)$ of chemisorbed water molecules (CWMs). A direct consequence of this observation is that each CWM induces an almost constant dipole moment independent of the $\mathrm{H}$ or water coverage. Figure 2(c) shows that $\Theta_{\mathrm{H}_{2} \mathrm{O}}$ rapidly decreases with $\mathrm{H}$ coverage. At $\Theta_{\mathrm{H}}=1 \mathrm{ML}$, the CWMs have almost completely disappeared. These observations highlight the central role of the CWMs on the electrode potential: They are the origin of the large downward shift of the electrode potential compared to the work function at low coverages and naturally explain why the two potentials become almost identical at full $\mathrm{H}$ coverage when CWMs cease to exist (Fig. 1).

To understand the nature of the large dipole moment with which the CWMs control the electrode potential, we inspect the difference charge density $\Delta n(\mathbf{r})$ due to water adsorption (Fig. 3) [58]. The analysis reveals that a CWM transfers part of an electron $(\approx 0.15 \mathrm{e})$ to the Pt surface, thus building up the above described large dipole moment. This observation resembles in many aspects the well-established Pt-water bonding model $[15,42,59]$ : the $\mathrm{H}_{2} \mathrm{O} 1 b_{1}$ lone pair orbital $\varepsilon_{\mathrm{O}\left(1 b_{1}\right)}$ strongly interacts with the $d$ orbitals $\varepsilon_{\mathrm{Pt}(d)}$ of the neighboring $\mathrm{Pt}$ atom [see Fig. 3(a)] giving rise to the formation of bonding-antibonding states (with energies $\varepsilon_{\mathrm{B}}$ and $\varepsilon_{\mathrm{AB}}$ ) [see Fig. 4(a)]. Conventionally, it is thought that $\varepsilon_{\mathrm{AB}}$ is above the Fermi level ( $\left.E_{\mathrm{Fermi}}\right)$, causing an electron transfer from the water molecule to the Pt surface atom below it $[60,61]$ [see Fig. 4(b)]. An analysis of the electron occupation of the respective $\mathrm{H}_{2} \mathrm{O}$ and $\mathrm{Pt}$ orbitals shows however a distinctly different behavior: While the $\mathrm{H}_{2} \mathrm{O}$ molecule indeed donates its electron and gains positive charge, the $\mathrm{Pt}$ atom to which the water molecule is

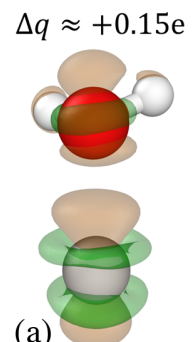

$\Delta q \approx+0.05 \mathrm{e}$
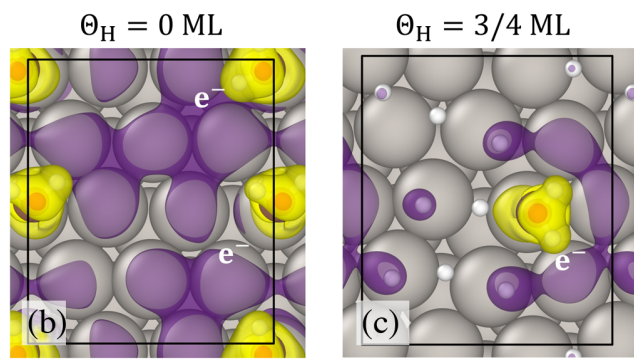

$\Delta q \approx-0.20$ e per CWM
FIG. 3. Difference charge density $\Delta n(\mathbf{r})$ represented as isosurfaces of (a) a CWM on top of a Pt atom and CWMs adsorbed on $\operatorname{Pt}\left(\begin{array}{lll}1 & 1 & 1\end{array}\right)$ with $\Theta_{\mathrm{H}}$ values of (b) $0 \mathrm{ML}$ and (c) $0.75 \mathrm{ML}$ sampled from the DFT-MD simulations (with the rest of the water molecules removed). $\Delta q$ is the approximate charge determined by integrating $\Delta n(\mathbf{r})$ in the vicinity of the atoms or molecules. The brown, green, yellow, and violet surfaces represent isolevels at $-0.036 \mathrm{e} / \AA^{3}, 0.036 \mathrm{e} / \AA^{3},-0.01 \mathrm{e} / \AA^{3}$, and $0.003 \mathrm{e} / \AA^{3}$, respectively (positive values represent electron accumulation). 
(a) $\uparrow$ Energy

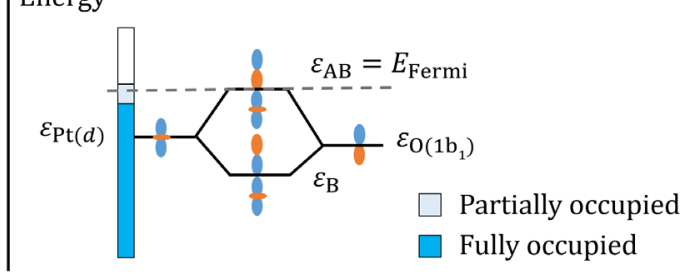

(b) Conventional model

(c) Revised model
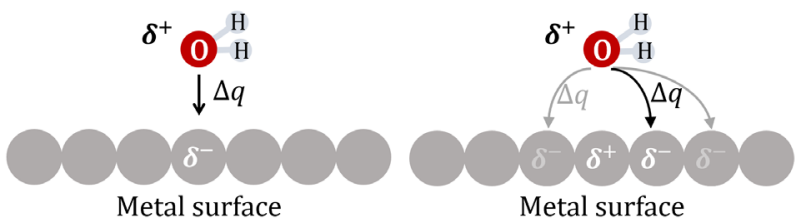

FIG. 4. Schematic representation of the interaction between metal surface and a CWM. (a) LCAO energy level diagram. (b) The conventional Pt-water bonding model with vertical charge transfer. (c) Derived model with diagonal transfer. The arrows in (b) and (c) indicate the transfer of fractional electrons to $\mathrm{Pt}$ atoms.

bonded also becomes positively charged $(\approx+0.05 \mathrm{e})$ [Fig. 3(a)].

A consequence of this finding is that the electron transfer is not vertical, i.e., from the on-top bonded water molecule to the Pt atom below, as commonly assumed [Fig. 4(b)]. Rather, both the water molecule and the on-top Pt atom act as electron donors, transferring a total of $\approx 0.2$ e per CWM [Figs. 3(b) and 3(c)]. Since a vertical transfer is blocked, a diagonal transfer to the $\mathrm{Pt}$ atoms neighboring the chemisorption site (with partially occupied states below $\varepsilon_{\mathrm{AB}}$ ) occurs [Fig. 4(c)]. This is evident in Fig. 3(b), where the violet regions show the orbitals into which the transfer occurs. Based on this insight, we conclude that the chemisorption of a CWM on a metal surface does not require only a single on-top $\mathrm{Pt}$ site but also neighboring $\mathrm{Pt}$ atoms to provide metallic orbitals for the diagonal charge transfer. Thus, a CWM requires a much larger surface area than originally thought, effectively reducing the number of available sites for adsorbates. Vice versa, adsorbates like $\mathrm{H}$ blocking these neighboring sites will prevent water chemisorption. This adsorbate induced blocking mechanism directly explains the observed anticorrelation between adsorbed $\mathrm{H}$ and CWMs [see Fig. 2(c)]. The decrease in the number of chemisorbed water molecules directly translates into the decrease in the magnitude of the electronic component $\Delta \Phi_{\text {elec }}$ of the electrode potential [Fig. 2(b)], which has been identified as the source of the voltage breakdown and thus the cause of instability for $\mathrm{H}$ adsorption above a critical coverage. The revised picture of the water-metal bond thus provides an intuitive explanation for the complex interplay between adsorbates and coadsorbed water molecules on metal surfaces.

A second interesting observation from the linear combination of atomic orbitals (LCAO) model shown in Fig. 4(a) is that the antibonding level of the CWM is pinned to the Fermi level of the metal. As a consequence, the CWM's highest occupied molecular orbital (HOMO) is partially rather than fully occupied. A partially occupied HOMO implies that the HOMO-LUMO (lowest unoccupied molecular orbital) gap of the CWM vanishes and that it is metallic. Because of their metallic character, the CWMs show a very different behavior in terms of screening, reactivity, etc. compared to conventional water molecules in the liquid.

In conclusion, we computed the electrode potential as a function of adsorbate coverage for one of the most studied reference systems in electrochemistry: $\mathrm{H}$ adsorbed on $\operatorname{Pt}\left(\begin{array}{lll}1 & 1 & 1\end{array}\right)$ in an aqueous environment employing extensive DFT-MD simulations. Based on a decomposition of the potential into the various contributions and by performing reference calculations for the same surfaces but in the absence of water we show that (i) water coadsorption and reorientation are relevant but (ii) at lower adsorbate coverages have opposite trends. The latter leads to a partial cancellation explaining why at these conditions the surface science picture works surprisingly well. At higher $\mathrm{H}$ coverages, however, the steady loss in chemisorbed water can no longer be compensated by water polarization, leading to an increase in electrode potential with increasing $\mathrm{H}$ coverage. A direct consequence of this phenomenon is that $\mathrm{H}$ adsorption above a critical $\mathrm{H}$ coverage becomes unstable, giving rise to desorption of the excess $\mathrm{H}$ that enables the hydrogen evolution reaction. The simulation protocols, the analysis concepts, and the insights derived in this study are general and can be straightforwardly applied to other electrochemical systems. The excellent agreement with available experimental data found in this study indicates that DFT calculations including explicit water provide an accurate tool to study complex structures and reactions at the solid-liquid electrochemical interface.

We thank Christoph Freysoldt and Stefan Wippermann for fruitful discussions. The authors acknowledge support from the RESOLV program by the Deutsche Forschungsgemeinschaft (DFG, German Research Foundation) under Germany's Excellence Strategy-EXC 2033-390677874-RESOLV and by the DFG through Project No. 405621160.

* Corresponding author. surendralal@mpie.de Corresponding author. m.todorova@mpie.de

[1] W. Schmickler and E. Santos, Interfacial Electrochemistry, 2nd ed. (Springer-Verlag, Berlin Heidelberg, 2010).

[2] N. M. Marković, B. N. Grgur, and P. N. Ross, J. Phys. Chem. B 101, 5405 (1997).

[3] N. M. Marković and P. N. Ross, Surf. Sci. Rep. 45, 117 (2002). 
[4] G. S. Karlberg, T. F. Jaramillo, E. Skúlason, J. Rossmeisl, T. Bligaard, and J. K. Nórskov, Phys. Rev. Lett. 99, 126101 (2007).

[5] Y. Li and M. J. Janik, Curr. Opin. Electrochem. 14, 124 (2019).

[6] K. Christmann, G. Ertl, and T. Pignet, Surface Sci. 54, 365 (1976).

[7] A. Hodgson and S. Haq, Surf. Sci. Rep. 64, 381 (2009).

[8] S. Maier and M. Salmeron, Acc. Chem. Res. 48, 2783 (2015).

[9] K. Thürmer and S. Nie, Proc. Natl. Acad. Sci. U.S.A. 110, 11757 (2013).

[10] O. M. Magnussen and A. Groß, J. Am. Chem. Soc. 141, 4777 (2019).

[11] S. Trasatti, J. Electroanal. Chem. Interfacial Electrochem. 209, 417 (1986).

[12] S. Trasatti, Electrochim. Acta 36, 1659 (1991).

[13] J. K. Nørskov, J. Rossmeisl, A. Logadottir, L. Lindqvist, J. R. Kitchin, T. Bligaard, and H. Jónsson, J. Phys. Chem. B 108, 17886 (2004).

[14] E. Skúlason, G. S. Karlberg, J. Rossmeisl, T. Bligaard, J. Greeley, H. Jónsson, and J. K. Nørskov, Phys. Chem. Chem. Phys. 9, 3241 (2007).

[15] A. Michaelides, V. A. Ranea, P. L. de Andres, and D. A. King, Phys. Rev. Lett. 90, 216102 (2003).

[16] J. Carrasco, A. Hodgson, and A. Michaelides, Nat. Mater. 11, 667 (2012).

[17] O. Björneholm, M. H. Hansen, A. Hodgson, L.-M. Liu, D. T. Limmer, A. Michaelides, P. Pedevilla, J. Rossmeisl, H. Shen, G. Tocci, E. Tyrode, M.-M. Walz, J. Werner, and H. Bluhm, Chem. Rev. 116, 7698 (2016).

[18] P. R. Norton and J. W. Goodale, Solid State Commun. 31, 223 (1979).

[19] S.-T. Cheng, M. Todorova, C. Freysoldt, and J. Neugebauer, Phys. Rev. Lett. 113, 136102 (2014).

[20] S. Nie, P. J. Feibelman, N. C. Bartelt, and K. Thürmer, Phys. Rev. Lett. 105, 026102 (2010).

[21] P. J. Feibelman, N. C. Bartelt, S. Nie, and K. Thrmer, J. Chem. Phys. 133, 154703 (2010).

[22] N. Dubouis and A. Grimaud, Chem. Sci. 10, 9165 (2019).

[23] Y. Zheng, Y. Jiao, A. Vasileff, and S.-Z. Qiao, Angew. Chem., Int. Ed. 57, 7568 (2018).

[24] J. Zheng, J. Nash, B. Xu, and Y. Yan, J. Electrochem. Soc. 165, H27 (2018).

[25] F. T. Wagner and T.E. Moylan, Surface Sci. 206, 187 (1988).

[26] T. J. Schmidt, P. N. Ross, and N. M. Marković, J. Electroanal. Chem. 524-525, 252 (2002).

[27] I. Hamada and Y. Morikawa, J. Phys. Chem. C 112, 10889 (2008).

[28] G. Jerkiewicz, Electrocatalysis 1, 179 (2010).

[29] T. Roman and A. Groß, Catal. Today 202, 183 (2013).

[30] F. Gossenberger, T. Roman, and A. Groß, Electrochim. Acta 216, 152 (2016).

[31] Y. Ishikawa, J. J. Mateo, D. A. Tryk, and C. R. Cabrera, J. Electroanal. Chem. 607, 37 (2007).

[32] J. J. Mateo, D. A. Tryk, C. R. Cabrera, and Y. Ishikawa, Mol. Simul. 34, 1065 (2008).

[33] J. A. Santana, J. J. Saavedra-Arias, and Y. Ishikawa, Electrocatalysis 6, 534 (2015).
[34] O. Sugino, J. Phys. Soc. Jpn. 89, 051013 (2020).

[35] H. H. Kristoffersen, T. Vegge, and H. A. Hansen, Chem. Sci. 9, 6912 (2018).

[36] S. Sakong and A. Groß, J. Chem. Phys. 149, 084705 (2018).

[37] G. Kresse and J. Hafner, Phys. Rev. B 47, 558 (1993).

[38] G. Kresse and J. Furthmüller, Phys. Rev. B 54, 11169 (1996).

[39] G. Kresse and J. Furthmüller, Comput. Mater. Sci. 6, 15 (1996).

[40] S. Surendralal, M. Todorova, M. W. Finnis, and J. Neugebauer, Phys. Rev. Lett. 120, 246801 (2018).

[41] See Supplemental Material, which includes Refs. [2,12, 35-40,42-55], at http://link.aps.org/supplemental/10.1103/ PhysRevLett.126.166802 for additional details.

[42] J. Le, M. Iannuzzi, A. Cuesta, and J. Cheng, Phys. Rev. Lett. 119, 016801 (2017).

[43] J. Neugebauer and M. Scheffler, Phys. Rev. B 46, 16067 (1992).

[44] G. Kresse and D. Joubert, Phys. Rev. B 59, 1758 (1999).

[45] H. J. Monkhorst and J. D. Pack, Phys. Rev. B 13, 5188 (1976).

[46] J. Janssen, S. Surendralal, Y. Lysogorskiy, M. Todorova, T. Hickel, R. Drautz, and J. Neugebauer, Comput. Mater. Sci. 163, 24 (2019).

[47] W. L. Jorgensen, J. Chandrasekhar, J. D. Madura, R. W. Impey, and M. L. Klein, J. Chem. Phys. 79, 926 (1983).

[48] J. P. Perdew, K. Burke, and M. Ernzerhof, Phys. Rev. Lett. 77, 3865 (1996).

[49] B. Hammer, L. B. Hansen, and J. K. Nørskov, Phys. Rev. B 59, 7413 (1999).

[50] S. Grimme, J. Antony, S. Ehrlich, and H. Krieg, J. Chem. Phys. 132, 154104 (2010).

[51] S. Sakong, K. Forster-Tonigold, and A. Groß, J. Chem. Phys. 144, 194701 (2016).

[52] V. Tripkovic, M.E. Björketun, E. Skúlason, and J. Rossmeisl, Phys. Rev. B 84, 115452 (2011).

[53] J. Le, A. Cuesta, and J. Cheng, J. Electroanal. Chem. 819, 87 (2018).

[54] J. Heyd, G. E. Scuseria, and M. Ernzerhof, J. Chem. Phys. 118, 8207 (2003).

[55] J. Heyd, G. E. Scuseria, and M. Ernzerhof, J. Chem. Phys. 124, 219906 (2006).

[56] The measurements are carried out using a $0.05 \mathrm{M} \mathrm{H}_{2} \mathrm{SO}_{4}$ solution with $p H=1.3$.

[57] It contains only the ionic component. The much smaller electronic component of the water polarization is already included in $\Delta \Phi_{\text {elec }}$.

[58] These results were confirmed by selected calculations using the hybrid HSE06 [54,55] functional (cf. Fig. 6 in the Supplemental Material).

[59] S. Meng, E. G. Wang, and S. Gao, Phys. Rev. B 69, 195404 (2004).

[60] T. Schiros, O. Takahashi, K. J. Andersson, H. Öström, L. G. M. Pettersson, A. Nilsson, and H. Ogasawara, J. Chem. Phys. 132, 094701 (2010).

[61] H. Ogasawara, B. Brena, D. Nordlund, M. Nyberg, A. Pelmenschikov, L. G. M. Pettersson, and A. Nilsson, Phys. Rev. Lett. 89, 276102 (2002). 